\title{
SATELLITE IMAGE RESOLUTION ENHANCEMENT USING MULTI WAVELET TRANSFORM AND COMPARISON OF INTERPOLATION TECHNIQUES
}

\author{
P.Suganya $^{1}$, N.Mohanapriya ${ }^{2}$, B.Kalaavathi ${ }^{3}$ \\ ${ }^{1} P G$ Student, CSE, Vivekanandha College of Engineering for Women, Namakkal, Tamilnadu, India \\ ${ }^{2}$ Assistant Professor, CSE, Vivekanandha College of Engineering for Women, Namakkal, Tamilnadu, India \\ ${ }^{3}$ Professor, CSE, K.S.R Institute for Engineering and Technology, Namakkal, Tamilnadu, India
}

\begin{abstract}
Abstract- The Researchers uses Satellite images for their Research work in many fields. The Satellite images have issues with their resolution. So, the images which loses their high frequency contents. And they appeared as blurred image. This paper proposes a resolution enhancement technique called Multi Wavelet Transform (MWT). This technique obtains the interpolation of high frequency sub bands and low resolution input image. The MWT uses Discrete Wavelet Transform (DWT) to split the image into sub bands. Then high frequency sub band images are obtained and the input images have been interpolated. Finally the inverse MWT have been applied to generate the enhanced high resolution satellite image. This paper analyses the performance of various image resolution techniques for satellite images. The proposed technique produces less artifacts with less Mean Square Error and increased values of Peak Signal to Noise Ratio.
\end{abstract}

Keywords: Image Resolution, Remote sensing, Multi wavelet Transform, Interpolation, Wavelet Zero Padding, Cycle Spinning

\section{INTRODUCTION}

Image processing is the processing of an image that converts input image into output image as either an image or set of characteristics. Then some useful information are extracted to get an enhanced image. Image processing is used in Remote Sensing, Medical Application, Graphics art and in many applications.

The Resolution of an image have to be increased in order to get an clear image. The linear features are found out by using the resolution. And this is also an important issue for satellite images. Many transforms are applied to various applications. Many existing transforms are basic for the development of the wavelet transform such as Discrete Wavelet Transform, Stationary Wavelet Transform, etc.

These remotely sensed image data is to be communicated from remote areas to receiver station, are facing with problems of storage and transmission of imagery data because of spatial resolution and it is limited by diffraction. Most of the satellites are operated on store-and-forward criterion; i.e. imagery have taken by satellites and stored. Then they are transmitted to ground stations. The transmissions have the drawback of storing data of high volumes. So, the satellite imagery systems have the increased demand on storage and require more time to transmit data. Satellite images are very useful for better understanding of the earth's condition. And they gives many sources of information for earth researchers. So there is a constant growth by remote sensing facilities which transmits the larger volume of data that have been processed efficiently.

\section{RELATED WORK}

The literature survey that describes different schemes available in resolution enhancement, the problem analysis of existing scheme and finally stated the proposed system

\subsection{The Image Sharing by Set Partitioning In Hierarchical Trees}

This says sharing information of an image by secret using multiwavelet transform It uses a set partitioning in hierarchical trees (SPIHT) code to improve the image quality and enhances processing of an image-sharing scheme. This paper[3] proposes two schemes are merging and splitting then rearranges the structures. The encoding process involves,

- A wavelet transform has applying to decompose an image and obtains coefficients.

- Then the coefficients are rearranged by the structure rearrangement process.

- SPIHT encoder uses the code for transform coefficients and converted to output binary bit stream.

- The bit-stream is shared by taking eight bits from the bit stream as generated in step 3, then the sharing number will be formed. 
The decoding process involves,

- $\quad$ Applying the SPIHT decoder.

- The inverse structure rearrangement process is applied to make the multiwavelet transform coefficients back to standard transform multi resolution representation.

- Finally the inverse multiwavelet transform is applied to revealed transform coefficients to reconstruct [3] the input image.

The method improves sharing method between images by secret and gives smaller shadows and good rate distortion performance.

\subsection{The Discrete Wavelet Transform method with}

\section{Adjacent Pixel Algorithm}

This have the process of Discrete Wavelet Transform in which the input image is splitted into different sub band images. Then interpolation process is applied to those sub band images. The low resolution image is enhanced by eliminating low pass filtering of high resolution image. And also low resolution image is used as input for further enhancement. The interpolation is done[7] with Adjacent Pixel Algorithm(APA). The APA in which takes the low sub band of an image and creates the empty column. Then computes the pixel value by averaging all pixel values. The high frequency sub bands contains clear view contents of an image.

Then the Inverse process of Discrete Wavelet Transform (IDWT) is applied to all sub band images to get a superresolved image. This method [7] preserves edge information.

\subsection{The Hybrid Directional Lifting Technique}

It describes the denoising of an image. Satellite images have affected by noise. The noise removing process from an image also have lossimg of high frequency contents. In order to give visual appearance and remove the noise, the (HDL) [15] technique is used.

This involves three steps:

- Pixel classification: It have two categories namely texture regions to classify the text and smooth regions to denoise an image.

- Orientation estimation: It provides the pixel estimation from pixel classification.

- Hybrid transform: It process the pixel level [15] and does not use the block level to reduce the noise.

The method denoises the image for an satellite image.

\subsection{Inter-Subband Correlation Technique using}

\section{Sub Bands}

It presents when an image is in low resolution, the image will be not clear that is denoted as blur image. Here the frequency will also be very low and discontinuous, so the property is shift variant due to frequency is not continuous, the property keeps on changing. If the resolution is high, the image will be clear because the frequency will be as much as continuous. The main advantage of using Inter-Sub band correlation is we can get continuous frequency, so the shifting property keeps invariant. The low resolution image will get clear and enhanced.

\subsection{The Dual-Tree Complex Wavelet Transform (DT-CWT) and Nonlocal Means (NLM) Filter}

The Dual-Tree Complex Wavelet Transform (DT-CWT) and Nonlocal Means (NLM) Filter which decompose the input image into sub bands and high frequency sub band images obtained. Then interpolation process is taken by combining high frequency sub bands and low resolution input image. Then those sub bands are passed through NLM filter. The NLM filter[9] which reduces the artifacts generated by input image. Then Inverse DT-CWT[9] process the combined filtered high frequency sub bands and input image. The technique which produces less artifacts

\section{PROPOSED WORK}

The proposed system includes Preprocessing, Apply Multi Wavelet Transform, Interpolation Process, Inverse Process of Multi Wavelet Transform for the low resolution image.

\subsection{Preprocessing}

The Haar (filter) features are used in recognizing the object. The features have similarity with Haar wavelets and they were used in real-time face detector to detect the object. A Haar filter processes the adjacent rectangular regions at particular location in a detection window which finds the pixel intensities in each region and sums up those intensities. The pixel intensities have the calculation of difference between sums. This difference is used to divide the subsections of an image.

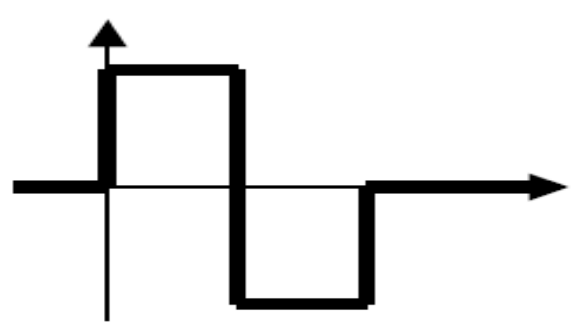

Fig.-1 Haar wavelet signal representation

In the one dimensional haar wavelet has the number of rows and columns and $\mathrm{A}$ denoted as Approximation, $\mathrm{H}$ denotes Horizontal, V denotes Vertical and D is Diagonal respectively.

The global properties information is carried out by approximation of analyzing images. $\mathrm{H}$ is horizontal area that describes information about vertical lines hidden in the image. $\mathrm{V}$ denotes vertical area that have information of 
horizontal lines hidden in the image. And D is diagonal area presents information of diagonal lines hidden in the image.

\subsection{Apply Multi Wavelet Transform}

The Multi Wavelet Transform (MWT) is applied and it is based on the interpolation of high frequency sub bands. The MWT uses Discrete Wavelet Transform (DWT) to decompose the image into various sub band images. The process obtains high frequency sub band images. Then interpolation process is taken by combining high frequency sub bands and input image which have low resolution. The inverse process of DWT is applied to produce a result image that have high resolution.

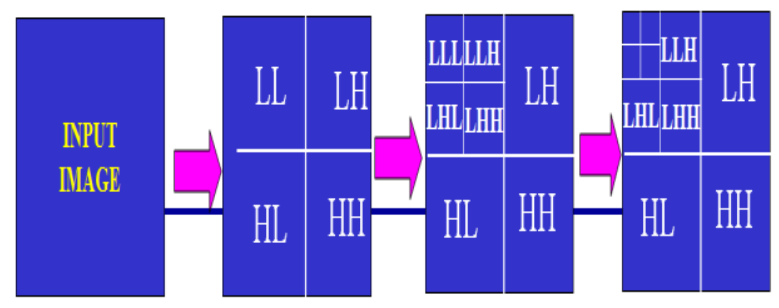

Fig.-2 Implementation of DWT

The DWT is the basic process and playing an important role in various image processing applications. The 1-D Multi Wavelet Transform take the rows of the image first and then results are processed along the columns. This leads to split the sub bands as low-low (LL), low-high (LH), high-low (HL), and high-high $(\mathrm{HH})$. So, the frequency sub bands cover the full original image. MWT has been proposed to get the clear image and have high frequency components.

\subsection{Interpolation Process}

It is the process of calculating the values of a function from the image samples. It have discrete input samples and the input values are evaluated at arbitrary positions in the input. The sampling process generates an infinite bandwidth signal and interpolation plays an opposite rule. It reduces this bandwidth signal by applying low pass filter.

This process used to estimate the pixel value and the noise will be reduced for an image. The estimate of a pixel at position $(p, q)$ is

$$
x(\mathrm{p}, \mathrm{q})=\frac{\sum_{\mathrm{m}=1}^{\mathrm{M}} \sum_{(\mathrm{r}, \mathrm{s}) \in \mathrm{N}(\mathrm{p}, \mathrm{q})} \operatorname{Ym}(r, s) K m(r, s)}{\sum_{\mathrm{m}=1}^{\mathrm{M}} \sum_{(r, s) \in N(p, q)} K m(r, s)}
$$

Where $m$ denotes frame index, and $N$ is the neighborhood of the pixel at location $(p, q)$ and $K$ denotes filter weights.

The interpolation process reduces signal lost by smoothing the data. This process is one of the fundamental operations in image processing. The image quality is derived by this interpolation process.

\subsection{Inverse Process Of Multi Wavelet Transform}

The interpolated high frequency sub band images and input low resolution image are combined. Then the difference image is used. The difference image is obtained by subtracting low sub band image from sub bands. And then interpolated low sub band image is combined. The unknown coefficients are replaced with zeros. The proposed technique have compared with existing technique, Discrete Wavelet Transform. And MWT produces less artifacts.

\section{EXPERIMENTAL RESULTS}

The input of Multi Wavelet Transform is Satellite Image with Low Resolution. And it have artifacts with noise.

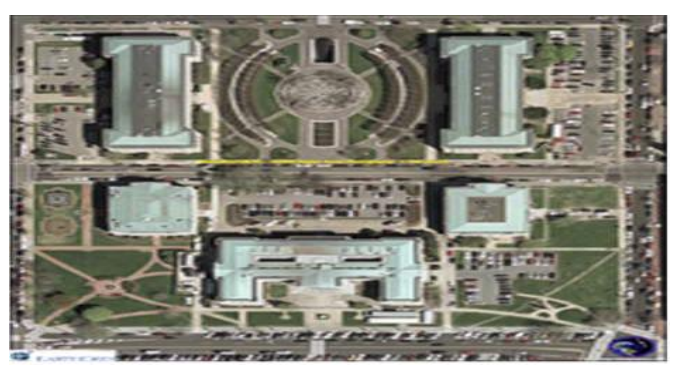

Fig.-3 (a) Input Image

The Multi Wavelet Transform which separates the image and during inverse process, it utilizes the difference image and is created by subtracting the input image and interpolated low sub band. And the peak signal to noise ratio, Mean Square Error values gets improved performance. Finally it produces high resolution image. The values are compared with interpolation techniques.

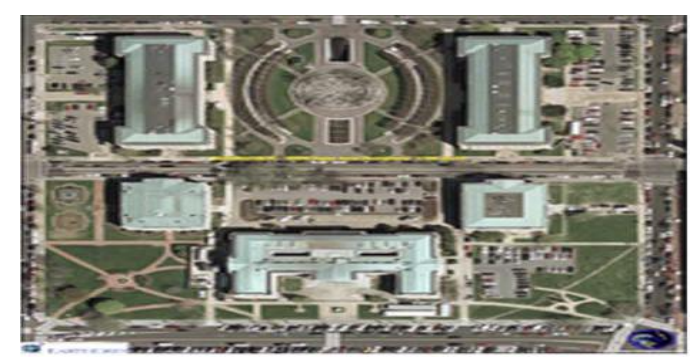

Fig.-3 (b) Output Image

\section{CONCLUSIONS}

Issues such as distortion, noise, artifacts, losing of high frequency contents have remained the most important challenges toward achieving resolution of satellite images. For improving the limitations of the above technique propose a resolution enhancement technique and it is based on the interpolation technique obtaining high frequency sub band images obtained by Discrete Wavelet Transform (DWT). The Multi Wavelet Transform and Interpolation technique which produces less artifacts. And also gives the performance interms of improved Peak Signal to Noise Ratio (PSNR) and reduced Mean Square Error (MSE). 


\section{REFERENCES}

[1]. [Online].Available:http://www.satimagingcorp.com

[2]. C. B. Atkins, C. A. Bouman, and J. P. Allebach, "Optimal image scaling using pixel classification," in Proc. Int. Conf. Image Process., Oct. 7-10, 2001, pp. 864-867.

[3]. Chin-Pan Huang and Ching-Chung Li "Secret Image Sharing Using Multiwavelet Transform" Journal of Information Science and Engineering 27, 733-748 (2011).

[4]. A.Gambardella and M.Migliaccio, "On the superresolution of microwave scanning radiometer measurements," IEEE Geosci. Remote Sens. Lett., vol. 5, no. 4, pp. 796-800, Oct. 2008.

[5]. M. Heng, 1. Chuanying and L. Shuang, "Multisource Image Fusion Based on Wavelet Transform", International Journal of Information Technology, Vol. 11, No.7, 2005.

[6]. Mahesh M and Venkata Srinu. M, "Low-Resolution satellite image enhancement using DT-CWT \& SVD” in International Journal of Advanced Research in Electronics and Communication Engineering (IJARECE), October 2012 , Volume 1, Issue 4, pp. 40-45.

[7]. E. Mohan, K.B. Jayarraman, U. Maheswaran, D. Sathiyaraj. G.Dhakshanamoorthi "A Novel Approach for Satellite Image Resolution Enhancement" , International Journal of Engineering and Advanced Technology (IJEAT) ISSN: 2249 - 8958, Volume-2, Issue-4, April 2013.

[8]. Muhammad Zafar Iqbal, Abdul Ghafoor, and Adil Masood Siddiqui "Satellite Image Resolution Enhancement Using Dual-Tree Complex Wavelet Transform and Nonlocal Means", IEEE Geoscience And Remote Sensing Letters, Vol. 10, No. 3, May 2013.

[9]. K.Narasimhan, V.Elamaran, Saurav Kumar, Kundan Sharma and Pogaku Raghavendra Abhishek, "Comparison of Satellite Image Enhancement Techniques in Wavelet Domain" in Research Journal of Applied Sciences, Engineering and Technology,2012, pp.1-5.

[10]. S. Nikolov, P.R. Hill, D.R. Bull, C.N. Canagarajah, "Wavelets in Signal and Image Analysis, from Theory to Practice", Kluwer Academic Publishers, 2001.

[11]. Parth Bhatt, Sachin Patel", Image Enhancement Using Various Interpolation Methods", International Journal of Computer Science and Information Technology \& Security (IJCSITS), ISSN: 2249-9555, Vol. 2, No.4, August 2012.

[12]. Y. Piao, I. Shin, and H. W. Park, "Image resolution enhancement using inter-subband correlation in wavelet domain," in Proc. Int. Conf. Image Process., San Antonio, TX, 2007, pp. I-445-I-448.

[13]. M. Protter, M. Elad, H. Takeda, and P. Milanfar, "Generalizing the nonlocal-means to super-resolution reconstruction,” IEEE Trans. Image Process., vol. 18, no. 1, pp. 36-51, Jan. 2009.

[14]. Rafael C Gonzalez and Richard E Woods, "Digital Image Processing", third edition, Pearson Education, 2007.

[15]. Sree Sharmila, T., Ramar, K. and SreeRenga Raja, T.

"Comparative Analysis Of Satellite Image Pre-Processing Techniques" Journal of Computer Science, 9 (2): 176-182, 2013 ISSN 1549-3636.

\section{BIOGRAPHIE}

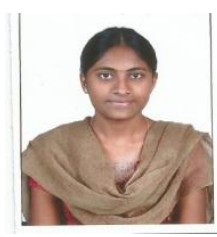

P.Suganya received B.E degree from Anna University, Chennai (Tamilnadu) in 2012. Currently she is pursuing M.E degree in Computer Science from Anna University, Chennai(Tamilnadu) Her research interest includes Image Processing . She has published papers in International Journals. 